\title{
Multiple Lanes Identification for Advanced Driver Assistance System (ADAS)
}

\author{
Suvarna Shirke, R. Udayakumar
}

\begin{abstract}
Now a days, a multi-lane recognition technique that uses the ridge features and the inverse perspective mapping (IPM) is generally used to distinguish lanes since it can evacuate the perspective distortion on lines that lie in parallel in reality. The lane detection is one of the approach to design the ADAS, if the vehicles follows the lane then there is less chance to get an accident. The detected information of lane path is used for controlling the vehicles and giving alerts to drivers. Therefore most of the researchers are attracted towards this field. But, due to the varying road conditions, it is very difficult to detect the lane. The computer vision and machine learning approaches are presents in most of the articles. In this paper, a survey of different method is presents for the road picture segmentation for the multi-lane detection. The Lane Departure Warning (LDW) system can help to reduce vehicle crashes that are caused by careless or drowsy driving. There has been much research on vision based lane detection for the $L D W$ system. In these lane detection methods, color or edge information is utilized as a feature of the lane. The feature-based methods are usually applied to localize the lanes in the road images by extracting low-level features. On the other hand, the model-based methods use several geometrical elements to describe the lanes, including parabolic curves, hyperbola and straight lines. Feature-based methods require a dataset containing several thousand images of the roads with well-painted and prominent lane markings that are subsequently converted to features. Moreover, these methods may suffer from noise.
\end{abstract}

Keywords :, Region Based Iterative Seed, Segmentation, Multilane Classification, Multilane Detection Advanced Driver Assistance System (ADAS).

\section{INTRODUCTION}

A dvanced driver assistance systems (ADAS) which either alert the driver in dangerous circumstances or take a functioning part in the driving, are continuously being embedded into vehicles. Such frameworks are relied upon to develop more and more complex towards full autonomy during the following decade. The primary problem in the improvement of such frameworks is the recognition issue, which has two components: street and path recognition, and snag (for example vehicles and passerby) location.

Road shading and surface, road limits and path markings are the fundamental perceptual signs for human driving.

Revised Manuscript Received on July 10, 2019

Suvarna Shirke, Research Scholar, Department of Computer Science and Engineering, Bharath Institute of Higher Education and Research, Chennai, India. Assistant Professor at Atharva College of Engineering.

Dr.R.Udayakumar, Professor and Supervisor, Department of Information Technology, Bharath Institute of Higher Education and Research, Chennai, India

Semi and completely self-sufficient vehicles are normal to impart the way to human drivers, and would therefore no doubt keep on depending on the equivalent perceptual signs people do. While there could be, on a basic level, different framework prompting for human drivers and vehicles (for example path marks for people and some type of vehicle-to-framework correspondence for vehicles) it is ridiculous to expect the tremendous ventures required to develop and keep up such twofold foundation, with the related hazard in bungled stamping. Street also, path discernment by means of the conventional signals remains in this way the no doubt way for self-sufficient driving.

For the machine learning and for computer vision the lane detection is the most hot topic which is applied to the intelligent vehicle systems[4]. It is very important to self-driving vehicles to identify the particular lane. These days, computer stereo vision has been predominantly used for improving the accuracy of the lane location system. Path recognition framework is utilized in different applications, as LDW, path bobbing identification, and blind spot checking. The line and edge detection is the main task for lane detection.

In recent years, lane detection is attracted by many researchers and many works had been implemented for detecting the lane[2]. Over the most recent couple of decades, several lane recognition methodologies are presented dependent on different sensors, similar to lidar, camera, and GPS. There are three steps of the lane detection system namely lane model fitting, feature extraction and tracking. Different systems, for example, color information, learning approaches, steerable filters, etc. are used for removing lane features by preparing the input pictures. Lane detection procedures utilizing hand-crafted features are utilized for identifying conventional shapes of markings and attempt to fit a spline or line for localizing lanes [7]. The ADAS system uses the cameras to track the lanes is used in most of the applications. This ADAS system gives notification to the driver if he is driving outside the lens unintentionally and takes automatic action to avoid wrong lane path. The secure driving tasks is rely upon the vehicle's driver. The position of a vehicle is depends on the road lane markings which helps to ADAS for the identification of vehicle. The presentation of path following depends on lighting conditions, path markings quality, and climatic components, similar to day off, and downpour. 
The plane of the road may perhaps vary in emergence due to neighboring trees, shadows casted by vehicles, tyre markings and construction material used [5]. Therefore it is more chances for wrong classification of getting the information of road markings. It is a challenge to identify the lane markings in the vehicle markings, also the lane marking sometimes completely disappears due to the heavy traffic conditions.

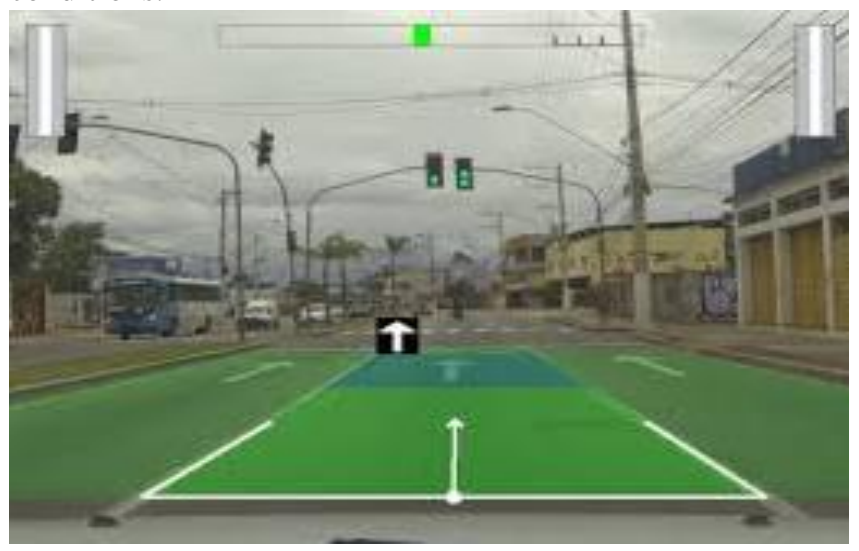

Fig. 1. Example of a Lane Detection.

It is also difficult to identify the side lanes due to the heavy traffic. Most of the papers presents the detection of ego lane instead of side lane. The traditional computer vision techniques are used for the most of lane detection in which a hand-made highlights are planned by difficult procedure offline tuning. This paper gives a few experiences in this area[8]. We present a forward-thinking review of methodologies and algorithms for street and path location as of late, updating the study from 2009. Figure 1 shows the example of lane detection.

The remainder of the work is sorted out as pursues: Section 1 is the introduction of proposed system. Segment 2 shows the proposed methods are used while designing this article. The evolutional matrices are presents in Segment 3. Section 4 shows the Experimental results of the proposed system. At last, the conclusion is presents in segment 5.

\section{LITERATURE SURVEY}

This section deals with the existing techniques of multi-lane detection system, explained as follows.

Apurba Das et al. [1] developed a binarization approach by modifying Min-Between-Max Thresholding (MBMT) approach, and hence, referred as MMBMT. The detection output of the developed method is considered to be prominent with global MBMT. The ground truth is determined as the base line frame, and thus, the minimum error can induce the drastic change in performance.

Soonhong Jung et al. [2] modelled a lane detection system using spatiotemporal images. Here, thelane points are detected by aligning scale lines with time axis and then, applying the Hough transform. The location of the detected lane points is utilized for computing the predicted alignment offset of the scanline in the next frame.

Heba Aly et al. [3] developed a lane detection model, commonly termed as LaneQuest. This framework is

introduced for detecting road lanes based on smart phone. Inappropriate cameras along with the smart phones are usedfor improving the probability of lane detection in extreme weather conditions.

Xinxin Du, and KokKiong Tan [4] presented effective stereo $3 \mathrm{D}$ reconstruction method for lane detection, and the model detected the lane using reconstruction method. This approach is essential for automated vehicle system owing to its high accuracy. Also, this scheme can be considered as the development to the vehicle lane-level localization. Some road features are considered as the lane markings, resulting in false detection.

Anti-shadow lane marking detection approach was developed by Zhenqiang Ying and Ge Li [5]. Here, road boundary detection techniques were introduced for extracting boundary information in the field of unstructured road detection. To detect lane markings, at first, road boundary information was extracted to build a road geometrical model, and then, lane markings were detected using Boundary-based IPM for Road Detection (BIRD). The method did not consider other methods for improving the road boundary segmentation based on boundary points fitting and Machine learning by RANSAC.

Yeongho Son et al. [6] developed a multi-lane detection and tracking approach for autonomous driving in a variety of environments on the highway. At first, adaptive threshold is employed for extracting strong lane features from images with obstacles and barely visible lanes. Then, the random sample consensus approach is established for preventing false lane detection. At last, the lane detection performance is enhanced by choosing only the lanes that are verified using classification approach.

Chao $\mathrm{Li}$ et al. [7] developed a robust and real-time multiple lane detection approach using Road Marking Feature Points (RMFP) for navigating autonomous vehicle in an urban environment. Here, RMFP is extracted based on IPM and gray-scale image. In addition, the structure features and the lane line color are utilized for sifting lane lines. After that, clustering technique is introduced for generating lane lines, and these lines are tracked using Kalman filter and frame association.

Jingchun Piao, and Hyunchul Shin [8] developed hypothesis generation technique for multiple lane detection based on binary blob, which is the development of top-view based approach. Hypothesis is generated based on Inverse Perspective Mapping (IPM) and Complement Laplacian of Gaussian Filter (cLoG) filter, and refined the binary blobs using blob verification and two-step blob filtering, followed by the hypothesis verification.

Several techniques are applied on lane detection, which can be categorized as model-based and feature-based [5]. The model based techniques present the lanes as a curve model that can be detected using an essential geometric parameter [7], whereas the feature based methods determine the lanes using low level features, like lane-mark edges [6].

Published By: 
Moreover, the feature based mechanisms are based on clear lane-marks, but suffer from weak lane marks, occlusions, and noise. The model based techniques are less susceptible for weak lanes features and noises as compared to feature based techniques. But, these models built for specific scene cannot work with another scene and makes the technique less adaptive. Moreover, an iterative error minimization algorithm is applied for analysing the model parameters in an optimal manner, but tends to be time consuming [8]. As compared to several techniques, the LDW systems based on machine takes automatic decisions for preventing the accidents with less cost and high reliability [9]. In perceptual systems, there exist different sensing modalities utilized for understanding road and lanes using monocular vision, Light Detection and Ranging (LIDAR) vehicle dynamics, and stereo and Global Positioning System (GPS). Vision is considered as the dominant research field for lane detection, while LIDAR and GPS are considered as the major complements [10]. For evaluating the lane model parameters, the Hough transform [12], chi-square fitting [13], and likelihood function [3] are utilized for detecting the lanes. But several lane models focus on specific shapes and lack flexibility for modelling the non-uniform road shape [14].

\section{CHALLENGES}

- A multi-lane detection method that uses the ridge feature and the inverse perspective mapping (IPM) is widely used to detect lanes because it can remove the perspective distortion on lines that lie in parallel in the real world [10]. The IPM transforms an image from a camera view to a bird's eye view by using camera parameters. Thus, lanes are presented perpendicularly and have the same width in the transformed image, where simple filters or geometric constraints can be used to detect lanes. In the IPM-based technique, however, the effectiveness of mapping is reduced if there are obstacles in the road [4].

- Methods using a vanishing point have been applied to lane detection. Since lanes are parallel to each other, they pass through their vanishing point in the image plane. Using this property, the false detections can be decreased by filtering out lines that do not pass through the vanishing point. Therefore, the vanishing point has been widely used to detect lanes [21] and roads. Furthermore, it can be used in various mobile applications where parallel lines detection plays an important role, such as corridor detection, power transmission lines inspection, 3D reconstruction, and so on. In order to extract a vanishing point exactly, first, lines should be extracted correctly. However, it is difficult to extract lines exactly from an image because of noises [4].

- A lane detection algorithm based on the B-Spline snake model. To initialize this model and track lanes, the vanishing point is estimated by using a Canny/Hough estimation of vanishing points (CHEVP). However, this method has a disadvantage of having a high false detection rate under shadowy or illuminated conditions [4].

- It's clearly understandable that, the binary images formed through NFGS, Otsu's and ABII cannot be used for the further process of ground truth generation as the left and right lane marks couldn't be extracted properly [2].

- Hough transform technique is used in the detection of lanes. However, their method has a drawback that the false positive rate can be high in an image with many spurious lines extracted from various safety markers on the road, from shadows, and so on [4].

\section{SCOPE OF PROBLEM}

The lane discovery issue, in any event in its fundamental setting, doesn't resemble a hard one. In this fundamental setting, one needs to recognize just the host lane, and just for a short distance ahead[3]. A generally basic Hough transform based calculation, which doesn't utilize any tracking or picture to-world thinking solves the problem in roughly $90 \%$ of the highway cases.

During the following decade, more and more semi-autonomous highlights are relied upon to be included slowly to vehicles, toward full autonomy. It can be effectively observed that Lane Departure Warning (LDW), the most essential of these features, has gotten the biggest part of research consideration. The path understanding level required for this element is identification of the host path alone, and to a separation of a few many meters ahead.

\section{A. Picture Clarity Issues:}

In most of the cases the road is clear and visible but sometimes due to the environmental condition it is very difficult to see road and due to this it can create an accident. The other problems like as shadows from close by trees, building material used and texture of the road also affect on this system[12]. Also due to the tunnel the improper illumination presents on the road.

\section{B. Poor Perceivability Conditions:}

The framework should work, or possibly distinguish the condition and lower its certainty, under downpour, mist, murkiness and night conditions. Each such condition requires another algorithmic treatment at some handling level.
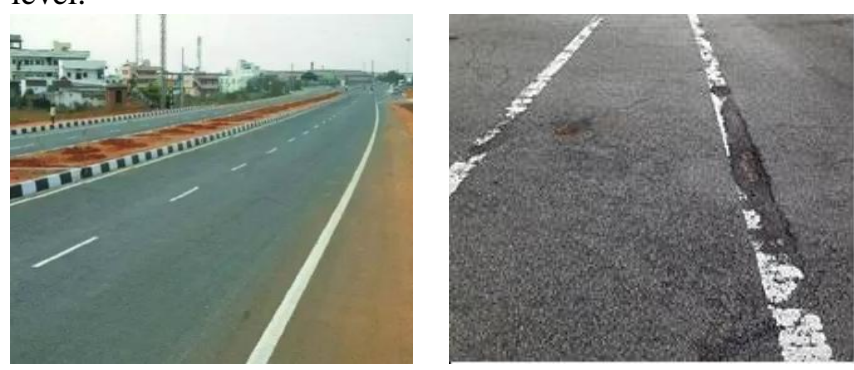

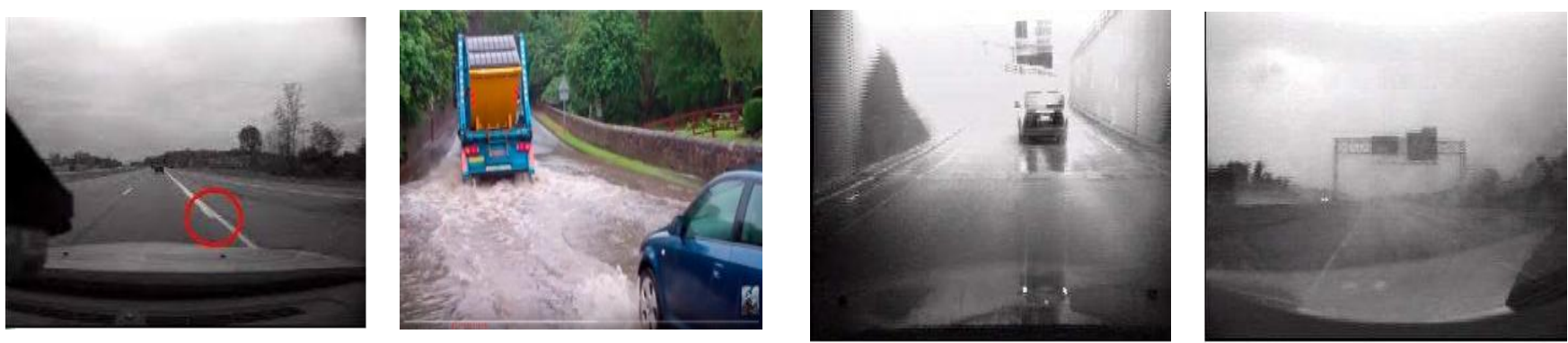

Fig. 2. Different Challenges for Lane Detection.

Table- II: Litrature Summery of Lane Detection System.

\begin{tabular}{|c|c|c|c|c|c|}
\hline Authors & Method Used & Features & Description & Advantage & Disadvantages \\
\hline $\begin{array}{l}\text { Son Lam } \\
\text { Phung [1] }\end{array}$ & $\begin{array}{l}\text { A vision-based } \\
\text { calculation for person on } \\
\text { foot path identification }\end{array}$ & 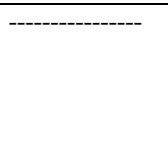 & $\begin{array}{l}\text { Person on foot } \\
\text { path identification } \\
\text { in unstructured } \\
\text { scenes for assistive } \\
\text { route }\end{array}$ & $\begin{array}{l}\text { exceptionally effective and } \\
\text { hearty as thought about } \\
\text { with a several existing } \\
\text { techniques. }\end{array}$ & $\begin{array}{l}\text { There are some division mistakes, where } \\
\text { there is a solid shadow }\end{array}$ \\
\hline $\begin{array}{l}\text { Heba Aly } \\
\text { et al. [2] }\end{array}$ & $\begin{array}{l}\text { An exact and energy } \\
\text { effective cell phone based } \\
\text { lane detection framework }\end{array}$ & $\begin{array}{l}\text { Powerful and } \\
\text { ubiquitous } \\
\text { cell phone } \\
\text { based lane } \\
\text { detection like } \\
\text { buildings, } \\
\text { parked cars, } \\
\text { etc. }\end{array}$ & $\begin{array}{l}\text { Cameras have } \\
\text { high energy } \\
\text { requirements for } \\
\text { the constrained } \\
\text { telephone battery }\end{array}$ & $\begin{array}{l}\text { This comes with a low-energy } \\
\text { footprint, allowing LaneQuest } \\
\text { to be implemented on the } \\
\text { energy-constrained mobile } \\
\text { devices. }\end{array}$ & $\begin{array}{l}\text { Using cameras for lane detection is highly } \\
\text { susceptible to errors due to various factors } \\
\text { such as lighting condition (e.g. night time, } \\
\text { sun glare, headlight glare, shadows from } \\
\text { nearby buildings, etc), bad weather } \\
\text { conditions (e.g. snow, rain), and other } \\
\text { environmental noise (e.g., faded lane } \\
\text { marks, surrounding objects. }\end{array}$ \\
\hline $\begin{array}{l}\text { Jianwei } \\
\text { Niu et al. } \\
{[3]}\end{array}$ & $\begin{array}{l}\text { Lane Detection with } \\
\text { Two-stage Feature } \\
\text { Extraction (LDTFE) }\end{array}$ & |---------------- & $\begin{array}{l}\text { Robust Lane } \\
\text { Detection using } \\
\text { Two-stage } \\
\text { Feature Extraction } \\
\text { with Curve Fitting }\end{array}$ & $\begin{array}{l}\text { can identify little line portions } \\
\text { situated on a straight line or a } \\
\text { line having little small } \\
\text { curvature. }\end{array}$ & $\begin{array}{l}\text { In any case, some nonexistent lines may } \\
\text { have a high vote an incentive due } \\
\text { to the nearness of numerous } \\
\text { non-consistent collinear edge pixels. In } \\
\text { this case, the discovery of short paths } \\
\text { turns out to be profoundly unlikely }\end{array}$ \\
\hline $\begin{array}{l}\text { Xinxin } \\
\text { Du, and } \\
\text { Kok Kiong } \\
\text { Tan [4] }\end{array}$ & $\begin{array}{l}\text { effective } \\
\text { stereo 3D reconstruction } \\
\text { method }\end{array}$ & $-\cdots$ & $\begin{array}{l}\text { Comprehensive } \\
\text { and Practical } \\
\text { Vision System for } \\
\text { Self-Driving } \\
\text { Vehicle } \\
\text { Lane-Level } \\
\text { Localization } \\
\end{array}$ & $\begin{array}{l}\text { Because of its high accuracy } \\
\text { and consistency, this framework } \\
\text { can be executed in independent } \\
\text { driving vehicles as a functional } \\
\text { solution for vehicle lane-level } \\
\text { localization. }\end{array}$ & $\begin{array}{l}\text { In any case, other than path line markings, } \\
\text { humps, zebra intersections, cautioning } \\
\text { letters and arrows also exhibit } \\
\text { comparative highlights and might be false } \\
\text { recognized as lane line markings }\end{array}$ \\
\hline $\begin{array}{l}\text { Ju Han } \\
\text { Yoo et al. } \\
{[5]}\end{array}$ & $\begin{array}{l}\text { a powerful path location } \\
\text { strategy based on } \\
\text { vanishing point } \\
\text { estimation }\end{array}$ & ----------------- & $\begin{array}{l}\text { A Robust Lane } \\
\text { Detection Method } \\
\text { Based on } \\
\text { Vanishing Point } \\
\text { Estimation Using } \\
\text { the Relevance of } \\
\text { Line Segments }\end{array}$ & $\begin{array}{l}\text { Efficiently estimates the } \\
\text { vanishing point and detects } \\
\text { lanes in different conditions }\end{array}$ & $\begin{array}{l}\text { The selected candidate line segments, } \\
\text { however, can contain line segments that } \\
\text { are extracted from the surrounding } \\
\text { environment and are not parallel to lanes } \\
\text { in the 3D space. }\end{array}$ \\
\hline $\begin{array}{l}\text { Soonhong } \\
\text { Jung et al. } \\
{[6]}\end{array}$ & $\begin{array}{l}\text { recognizing street paths } \\
\text { dependent on } \\
\text { spatiotemporal pictures }\end{array}$ & $\begin{array}{ll}---- \\
\end{array}$ & $\begin{array}{l}\text { Productive Lane } \\
\text { Detection Based } \\
\text { on Spatiotemporal } \\
\text { Images }\end{array}$ & $\begin{array}{l}\text { computation times reduced to as } \\
\text { little as one-third as compared } \\
\text { to other methods }\end{array}$ & $\begin{array}{l}\text { just a somewhat improved pace of } \\
\text { identification. }\end{array}$ \\
\hline $\begin{array}{l}\text { Apurba } \\
\text { Das et al. } \\
{[7]}\end{array}$ & $\begin{array}{l}\text { A novel binarization } \\
\text { calculation dependent on } \\
\text { min-between-max } \\
\text { thresholding (MBMT). }\end{array}$ & ----- & $\begin{array}{lr}\text { Improved } \\
\text { Algorithm } \\
\text { Automated } \\
\text { Ground } \\
\text { Generation } \\
\text { Validation and } \\
\text { Lane Detection } \\
\text { System } \\
\text { M2BMT } \\
\end{array}$ & $\begin{array}{l}\text { the path markings distinguished } \\
\text { are seen to be significantly } \\
\text { prominent as for traditional } \\
\text { global MBMT }\end{array}$ & $\begin{array}{l}\text { As the ground truth casing work would be } \\
\text { utilized as a pattern edge to be considered } \\
\text { as reference at the hour of approval, even } \\
\text { a little blunder wouldn't be satisfactory. } \\
\text { Subsequently the calculation ought to } \\
\text { likewise coordinate the client with edges } \\
\text { where manual mediation is required. }\end{array}$ \\
\hline $\begin{array}{l}\text { Umar } \\
\text { Ozgunalp } \\
\text { et al. }[8]\end{array}$ & $\begin{array}{l}\text { novel path location } \\
\text { calculation }\end{array}$ & ------ & $\begin{array}{lr}\text { Different } & \text { Lane } \\
\text { Detection } & \\
\text { Algorithm } & \text { Based } \\
\text { on Novel } & \text { Dense } \\
\text { Vanishing } & \text { Point } \\
\text { Estimation } & \end{array}$ & $\begin{array}{l}\text { can identify different paths with } \\
\text { both flat and vertical shape, } \\
\text { works vigorously and precisely } \\
\text { even in thick rush hour } \\
\text { gridlock. }\end{array}$ & $\begin{array}{l}\text { The experimental set-up is physically } \\
\text { introduced onto the vehicle utilizing } \\
\text { air-suction cushions and a lot of move } \\
\text { edge is should have been acquainted due } \\
\text { with this underlying establishment. } \\
\text { Despite the fact that the move point } \\
\text { doesn't change altogether after some time, } \\
\text { the move edge presented during camera } \\
\text { establishment onto the vehicle should be } \\
\text { assessed as a piece of the alignment } \\
\text { procedure. }\end{array}$ \\
\hline
\end{tabular}




\begin{tabular}{|c|c|c|c|c|c|}
\hline $\begin{array}{l}\text { Rasmusse } \\
\text { n, C., } \\
\text { Korah, } \\
\text { T[9] }\end{array}$ & $\begin{array}{l}\text { Full self-sufficient } \\
\text { driving for cross country } \\
\text { driving }\end{array}$ & $\begin{array}{l}\text { Self-sufficien } \\
\mathrm{t} \text { driving in } \\
\text { non-cleared } \\
\text { areas }\end{array}$ & $\begin{array}{l}\text { On-vehicle and } \\
\text { airborne surface } \\
\text { investigation for } \\
\text { vision-based } \\
\text { desert street } \\
\text { following. In: } \\
\text { CVPR workshop } \\
\text { on machine vision } \\
\text { for savvy vehicles. }\end{array}$ & $\begin{array}{l}\text { Full unpleasant street seeing } \\
\text { however fairly simpler than } \\
\text { cleared street autonomy as for } \\
\text { absence of paths, sparser traffic }\end{array}$ & |-------------- \\
\hline $\begin{array}{l}\text { Gao, T., } \\
\text { Aghajan, } \\
\text { H[11] }\end{array}$ & $\begin{array}{l}\text { Self path task utilizing } \\
\text { egocen-tric keen versatile } \\
\text { camera for smart GPS } \\
\text { route }\end{array}$ & $\begin{array}{l}\text { Path change } \\
\text { help }\end{array}$ & $\begin{array}{l}\text { Self-governing } \\
\text { turn on driver } \\
\text { demand or as a } \\
\text { feature of } \\
\text { programmed route }\end{array}$ & $\begin{array}{l}\text { Various paths, path semantics } \\
\text { (identify turning paths), } \\
\text { non-straight path and street } \\
\text { topology (parts } \\
\text { consolidations) }\end{array}$ & |-------------" \\
\hline $\begin{array}{l}\text { Jiang, Y., } \\
\text { Gao, F., } \\
\text { Xu, G [12] }\end{array}$ & $\begin{array}{l}\text { PC vision-put together } \\
\text { numerous path } \\
\text { recognition with respect } \\
\text { to straight street and in a } \\
\text { bend }\end{array}$ & Turn help & $\begin{array}{l}\text { Independent path } \\
\text { change on request }\end{array}$ & |--------------- & ------------- \\
\hline $\begin{array}{l}\text { Wu, S., } \\
\text { Chiang, } \\
\text { H., Perng, } \\
\text { J., Chen, } \\
\text { C.,Wu, B., } \\
\text { Lee, T[13] }\end{array}$ & $\begin{array}{l}\text { The heterogeneous } \\
\text { frameworks coordination } \\
\text { structure and execution } \\
\text { for path keeping on a } \\
\text { vehicle }\end{array}$ & Path focusing & $\begin{array}{l}\text { Keep the vehicle } \\
\text { in the path } \\
\text { consistently }\end{array}$ & |--------------- & |------------- \\
\hline $\begin{array}{l}17] \\
\text { Hofmann, } \\
\text { U., Rieder, } \\
\text { A., } \\
\text { Dickmann } \\
\text { s[14] }\end{array}$ & $\begin{array}{l}\text { Radar and vision } \\
\text { information combination } \\
\text { for half and half versatile } \\
\text { journey control on } \\
\text { expressways }\end{array}$ & $\begin{array}{l}\text { Adaptive } \\
\text { Cruise } \\
\text { Control } \\
\text { (ACC) }\end{array}$ & $\begin{array}{l}\text { Pursue the closest } \\
\text { vehicle in the host } \\
\text { path with safe } \\
\text { head- } \\
\text { separation way }\end{array}$ & |--------------- & |--------------- \\
\hline $\begin{array}{l}\text { Batavia, } \\
\text { P.H[15] }\end{array}$ & $\begin{array}{l}\text { Driver-versatile path } \\
\text { takeoff cautioning sytems }\end{array}$ & $\begin{array}{l}\text { Lane } \\
\text { Departure } \\
\text { Warning } \\
\text { (LDW) } \\
\end{array}$ & $\begin{array}{l}\text { Issue alerts for } \\
\text { close to path } \\
\text { departure } \\
\text { occasions }\end{array}$ & |--------------- & |--------------- \\
\hline
\end{tabular}

\section{DISCUSSION}

While capturing the road lane picture, there are lots of difficulties are occurs. These difficulties are occurs due to the condition of road. Figure 2 shows some of the examples which affects on the road lane condition. This can be happen due to the different environment condition, low quality of material used while construction of road, due to the fog, different lighting conditions, etc. Also very less volume of iris is captured due to the small size of iris, camera diffraction, acquisition distance, human machine interference, etc. To avoid this types of problems much many techniques are designed. The techniques which are used to solve these problems are use moderate hardware system , reduces the noise effects and other distortions, focus to increase the quality of input picture, use of larger megapixel cameras, Wave front Coding, use of telescope, etc. Figure 3 shows the different lines on the road. Most of the images captured by camera are noisy, so it is very difficult to identify the correct lane for the boundary-detection algorithms.

These days, computer stereo vision has been predominantly used for improving the accuracy of the lane location system. Table 1 shows the review summary of different lane detection systems in terms of used methods, feature, description, advantages and disadvantages, etc. There are fifteen papers are considered for the literature review and near about eight different methods are presents in this. Table 2 shows the comparative discussion of different methods in terms of detection accuracy, sensitivity and specificity. From this table it is clear that the accuracy of the methods used is more than the other parameter.

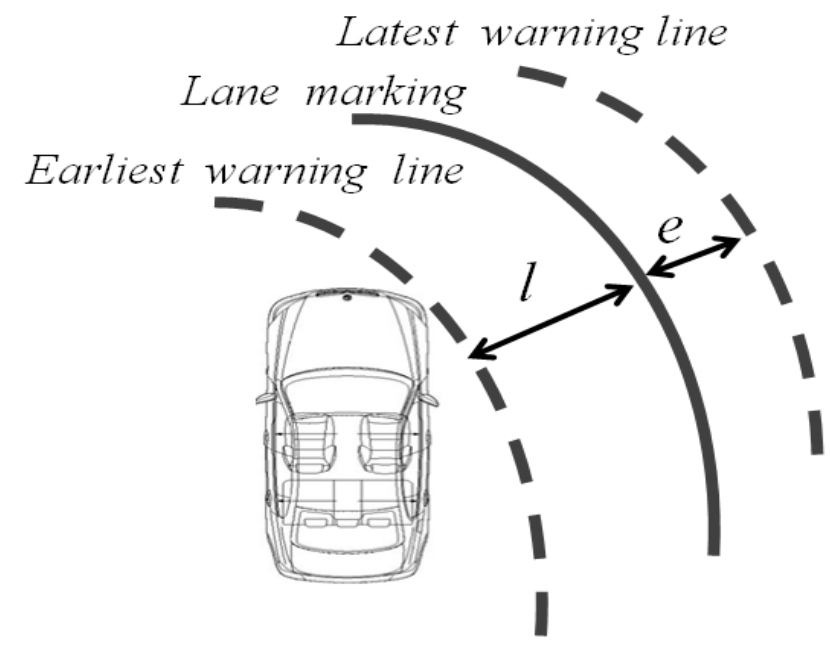

Fig. 3. Different Lines on the Road. 


\section{CONCLUSION}

Advanced Driver Assistance System (ADAS) for the multi-lane detection is used in large amount of real time applications. In this article, different methods review is present to solve the complexity of road understanding problems. For example non linear topologies of road and lane ,perception of multiple lanes, noises due to different lighting condition, etc. The pointless foundation pictures are additionally expelled with the assistance of picture division process. Some of the improvements are improved segmentation and feature extraction, quality enhancement and classification. As compared to steady objects, the acquisition of picture of moving object. Also, many problems of iris recognition were solved like as human-machine interface, image acquisition, and image processing problems.

Table II Comparative discussion

\begin{tabular}{|c|c|c|c|}
\hline Methods & $\begin{array}{c}\text { Detection } \\
\text { Accuracy }\end{array}$ & Sensitivity & Specificity \\
\hline DVPE & 0.9141 & 0.8712 & 0.74 \\
\hline $\begin{array}{c}\text { EW-CSA based } \\
\text { DCNN }\end{array}$ & 0.9785 & 0.9788 & 0.7655 \\
\hline DCNN & 0.9838 & 0.9762 & 0.7041 \\
\hline MMBM & 0.9107 & 0.9417 & 0.7440 \\
\hline $\begin{array}{c}\text { Region based } \\
\text { Iterative Seed }\end{array}$ & 0.9889 & 0.9916 & 0.8863 \\
\hline
\end{tabular}

\section{ACKNOWLEDGMENT}

We are grateful to the Department of Computer Science and Engineering, Bharath Institute of Higher Education and Research, Chennai, India. for allowing to utilize the computational offices by giving us most reasonable condition to this innovative work.

\section{REFERENCES}

1. Son Lam Phung, Manh Cuong Le , and Abdesselam Bouzerdoum, "Pedestrian lane detection in unstructured scenes for assistive navigation", Computer Vision and Image Understanding, vol.149, pp.186-196, 2016.

2. Heba Aly, Anas Basalamah, and Moustafa Youssef, "Robust and ubiquitous smartphone-based lane detection", Pervasive and Mobile Computing, 2015.

3. Jianwei Niu, JieLu, Mingliang Xu , PeiLv, and Xiaoke Zhao, "Robust Lane Detection using Two-stage Feature Extraction with Curve Fitting", Pattern Recognition, vol 59, pp.225-233, November 2016.

4. Xinxin Du, and Kok Kiong Tan, "Comprehensive and Practical Vision System for Self-Driving Vehicle Lane-Level Localization", IEEE Transactions On Image Processing, Vol. 25, No. 5, May2016.

5. Ju Han Yoo, Seong-Whan Lee, Sung-Kee Park, and Dong Hwan Kim, "A Robust Lane Detection Method Based on Vanishing Point Estimation Using the Relevance of Line Segments", IEEE Transactions On Intelligent Transportation Systems", vol.18, no.12, February 2017.

6. Soonhong Jung, Junsic Youn, and Sanghoon Sull, "Efficient Lane Detection Based on Spatiotemporal Images", IEEE Transactions On Intelligent Transportation Systems, January 2015.

7. Apurba Das, Member, Siva Srinivasa Murthy, and Upendra Suddamalla, "Enhanced Algorithm of Automated Ground Truth Generation and Validation for Lane Detection System by M2BMT", IEEE Transactions On Intelligent Transportation Systems, Vol. 18, No. 4, April 2017

8. Umar Ozgunalp, Rui Fan, Xiao Ai, and Naim Dahnoun, "Multiple Lane Detection Algorithm Based on Novel Dense Vanishing Point Estimation", Ieee Transactions On Intelligent Transportation Systems, Vol. 18, No. 3, March 2017

9. Rasmussen, C., Korah, T.: On-vehicle and aerial texture analysis for vision-based desert road following. In: CVPR workshop on machine vision for intelligent vehicles. (2005) III: $66\{66$

10. Montemerlo, M., et al.: Junior: The Stanford entry in the Urban Challenge. Journal of Field Robotics 25(8) (2008) 569\{597 25
11. Gao, T., Aghajan, H.: Self lane assignment using egocen- tric smart mobile camera for intelligent GPS navigation. In: Workshop on Egocentric Vision. (2009) $57\{62$

12. Jiang, Y., Gao, F., Xu, G.: Computer vision-based multiple- lane detection on straight road and in a curve. In: ImageAnalysis and Signal Processing. (2010) $114\{117$

13. Wu, S., Chiang, H., Perng, J., Chen, C.,Wu, B., Lee, T.: The heterogeneous systems integration design and implementation for lane keeping on a vehicle. IEEE Transactions on Intelligent Transportation Systems 9 (2008) $246\{263$

14. Hofmann, U., Rieder, A., Dickmanns, E.: Radar and vision data fusion for hybrid adaptive cruise control on highways. Machine Vision and Applications 14 (2003) 1, 42\{49

15. Batavia, P.H.: Driver-adaptive lane departure warning sytems CMU-RI-TR-99-25 (1999)

16. Meng-Che Chuang, Jenq-Neng Hwang, and Kresimir Williams", A Feature Learning and Object Recognition framework for underwater fish images", IEEE transactions on image processing, vol.25, no.4, pp.1862-1872, 2016.

17. Yuichi Saito, Makoto Itoh, and Toshiyuki Inagaki,"Driver Assistance System with a Dual Control Scheme: Effectiveness of Identifying Driver Drowsiness and Preventing Lane Departure Accidents",IEEE transactions on human-machine systems, March 2016.

18. K. Bengler, K. Dietmayer, B. Farber, M. Maurer, C. Stiller, and H. Winner, "Three decades of driver assistance systems: Review and future perspectives," IEEE Intelligent Transportation Systems Magazine, vol. 6, no. 4, pp. 6-22, April 2014.

19. Ju Han Yoo, Seong-Whan Lee, Sung-Kee Park, and Dong Hwan Kim, "A Robust Lane Detection Method Based on Vanishing Point Estimation Using the Relevance of Line Segments", IEEE Transactions On Intelligent Transportation Systems", vol.18, no.12, February 2017.

20. Gurveen Kaur, and Dinesh Kumar,"Lane Detection Techniques: A Review", International Journal of Computer Applications, vol.112, no 10, pp.0975 - 8887, February 2015.

21. Seokju Lee, Junsik Kim, Jae Shin Yoon, Seunghak Shin,OleksandrBailo, Namil Kim Tae-Hee Lee, Hyun Seok Hong, Seung-Hoon Han, and In So Kweon, "VPGNet: Vanishing Point Guided Network for Lane and Road MarkingDetection and Recognition", In Proceedings of the IEEE International Conference on Computer Vision, pp. 1947-1955, 2017.

22. Alexander Rakhlin, Alexey Shvets, Vladimir Iglovikov, and Alexandr A. Kalinin, "Deep Convolutional Neural Networks for Breast Cancer Histology Image Analysis", In proceedings of International Conference on Image Analysis and Recognition ICIAR, Image Analysis and Recognition, pp. 737-744, 2018.

23. Jun Li, XueMei, Danil Prokhorov, and Dacheng Tao,"Deep Neural Network for Structural Predictionand Lane Detection in Traffic Scene",IEEE transactions on neural networks and learning systems, January 2016.

24. Mane, V.M. and Jadhav, D.V., "Holoentropy enabled-decision tree for automatic classification of diabetic retinopathy using retinal fundus images," Biomedical Engineering/Biomedizinische Technik, vol.62, no.3, pp.321-332, 2017

25. The KITTI Vision Benchmark Suite taken from, "http://www.cvlibs.net/datasets/kitti/eval_road.php", accessed on August 2018.

26. Umar Ozgunalp, Rui Fan, Xiao Ai, and NaimDahnoun, "Multiple Lane Detection Algorithm Based on Novel Dense Vanishing Point Estimation", IEEE Transactions On Intelligent Transportation Systems, Vol. 18, No. 3, March 2017.

\section{AUTHORS PROFILE}

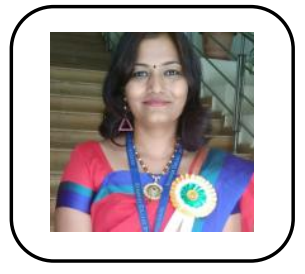

Suvarna Shirke-Pansambal is the Research Scholar at Bharath Institute of Higher Education and Research, Department of Computer Science \& Engineering, Chennai. She is working as an Assistant Professor in Atharva College of Engineering, Mumbai.

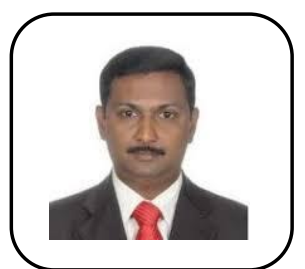

Dr.R.Udayakumar is Professor at Department of Information Technology, Bharath Institute of Higher Education and Research, Chennai, India. He has published more than 600 papers and he is Supervisor for Research Scholars at BIHER. 